\title{
COVID-19 effects on neuro-oncology publishing: preliminary outcomes \& future impacts
}

\author{
Christopher P. Cifarelli ${ }^{1}\left[\right.$ ] Jason P. Sheehan ${ }^{2}$
}

Received: 30 August 2020 / Accepted: 10 September 2020 / Published online: 26 September 2020

○) Springer Science+Business Media, LLC, part of Springer Nature 2020

\section{To the Editor,}

The true impact of COVID-19 on the global healthcare delivery system will likely not be fully realized for years to come. Within our own sub-specialties of medicine, the pandemic has forced providers and patients to adjust to the new "normal" that came with social distancing, contact tracing, and for some, the brief, but impactful, cessation of elective surgical procedures. In most centers, neuro-oncology remained a priority for timely care delivery and the "elective pause" did not apply, but it would be rare to identify a single practice that did not feel the impact in some way [1].

With regard to the academic pursuits of those engaged in the practice of neuro-oncology, the pandemic had the real capacity to negatively impact our progress. Many systems temporarily halted new patient enrollment in clinical trials, while most universities provided strict guidelines on conducting basic science research, marshaling resources and prioritizing work that directly focused on COVID-19 related areas $[2,3]$. Now, as we have passed into the third quarter of 2020 and plans for a return to "normal" activity levels continue to expand, we can begin to quantify the effect that the pandemic and the associated responses have had on our field, specifically with respect to medical publishing.

Since 2017, the Journal of Neuro-oncology has seen a steady increase in new submissions, ranging from 18 to $23 \%$ year-over-year through 2019 (Fig. 1b). Over this period, three-quarters of the annual submissions were based on clinical studies with the remaining $25 \%$ representing laboratory investigations (Fig. 1c). Through the first and second quarters of 2020, we have witnessed a near doubling of the

Christopher P. Cifarelli

cpcifarelli@hsc.wvu.edu

1 Departments of Neurosurgery \& Radiation Oncology, West Virginia University, Morgantown, WV, USA

2 Department of Neurosurgery, University of Virginia, Charlottesville, VA, USA new submissions, with a rate increase of $44 \%$ from 2019 for the same period, maintaining the same mixture of clinical and laboratory studies (Fig. 1b, c).

As the JNO serves a multi-disciplinary, international readership, we are cognizant of the differences in COVID19 rates across different nations along with differences in strategies used to aid in dealing with the virus. A review of the submission rate changes from 2019 through Q1-Q2 of 2020 based on selected countries of origin demonstrated increases ranging from 7 to $112 \%$, with only submissions from Japan experiencing a modest decline (Fig. 1a).

Although assigning causation to the steep increase in JNO submission rates would be highly presumptive, we can speculate that the changes in primary practice patterns among our author pool may have afforded more time to engage in manuscript preparation or revision than during the previous years evaluated. The generation of the high quality manuscripts received by the JNO remains a time-intensive process that, under normal clinical demand conditions, could be deferred. Yet, the reduction in travel for meetings, vacations, and general daily activities during the COVID-19 pandemic may have contributed to more time available for manuscript preparation.

As we have mentioned in our opening statement, the scope of impact of COVID-19 on our personal and professional lives remains undetermined. In our current analysis, the submission rate for the third and fourth quarters of 2020 will remain critically important. Increased clinical demands as healthcare systems return to full capacity could have the ability to swing the pendulum in the other direction, but thus far into the third quarter, the increase in submissions has continued. Moreover, the basic science laboratories have experienced an even slower repatriation in most centers, opening the possibility of a substantial lapse in the time to acquire new data for publication. Regardless of the obstacles or outcomes, the JNO remains dedicated to the goal of providing our readership the highest quality publications in the field of Neuro-oncology and offering our authors the 
A

\begin{tabular}{c|c} 
& $\%$ Change \\
\hline USA & 48 \\
\hline CHINA & 7 \\
\hline JAPAN & -23 \\
\hline GERMANY & 32 \\
\hline CANADA & 70 \\
\hline FRANCE & 31 \\
\hline ITALY & 112 \\
\hline INDIA & 87
\end{tabular}

B

\section{Annual Submissions}

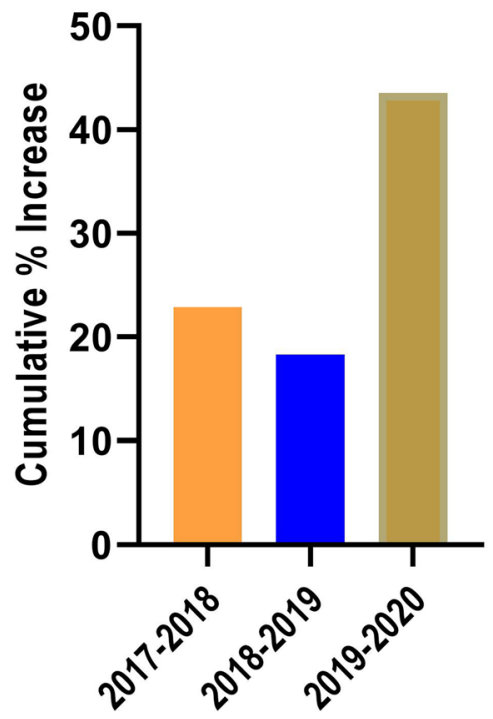

\section{C}

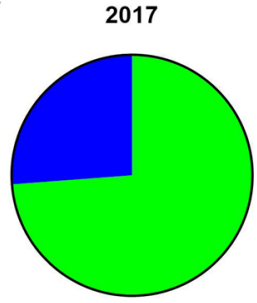

2018

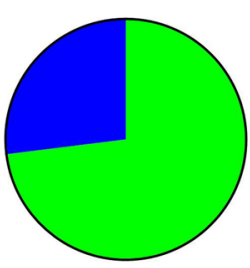

2019

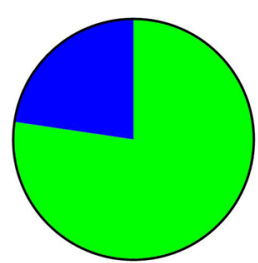

2020

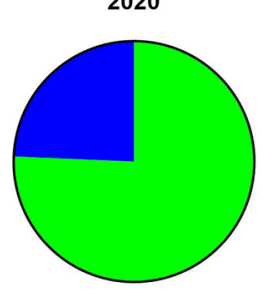

Clinical Study

Laboratory Investigation
Fig. 1 JNO Submission Trends, 2017 to 2020. The change in submission rate from 2019 to 2020 based on selected countries of origin (a) and overall rate increases (b) demonstrate an upward trend beyond that seen prior to COIVD-19, while maintaining the same mixture of clinical and laboratory investigations (c)

neuro-oncology practitioners from the AANS/CNS Tumor Section and Society for Neuro-Oncology. J Neurooncol 147:525-529. https://doi.org/10.1007/s11060-020-03488-7

2. Servick K, Cho A, Guglielmi G, Vogel G, Couzin-Frankel J (2020) Updated: labs go quiet as researchers brace for long-term coronavirus disruptions. Science 16 March 2020

3. Ledford $\mathrm{H}$ (2020) Coronavirus shuts down trials of drugs for multiple other diseases Nature 580:15-16

Publisher's Note Springer Nature remains neutral with regard to jurisdictional claims in published maps and institutional affiliations. 